\title{
An Unsupervised Approach for Acquiring Ontologies and RDF Data from Online Life Science Databases
}

\author{
Saqib Mir ${ }^{1,2}$, Steffen Staab $^{2}$, and Isabel Rojas ${ }^{1}$ \\ 1 EML-Research Schloss-Wolfsbrunnenweg 31c, 69118 Heidelberg, Germany \\ 2 University of Koblenz-Landau, Koblenz, Germany \\ \{saqib.mir,isabel.rojas\}@eml-r.org, staab@uni-koblenz.edu
}

\begin{abstract}
In the Linked Open Data cloud one of the largest data sets, comprising of 2.5 billion triples, is derived from the Life Science domain. Yet this represents a small fraction of the total number of publicly available data sources on the Web. We briefly describe past attempts to transform specific Life Science sources from a plethora of open as well as proprietary formats into RDF data. In particular, we identify and tackle two bottlenecks in current practice: Acquiring ontologies to formally describe these data and creating "RDFizer" programs to convert data from legacy formats into RDF. We propose an unsupervised method, based on transformation rules, for performing these two key tasks, which makes use of our previous work on unsupervised wrapper induction for extracting labelled data from complete Life Science Web sites. We apply our approach to 13 real-world online Life Science databases. The learned ontologies are evaluated by domain experts as well as against gold standard ontologies. Furthermore, we compare the learned ontologies against ontologies that are "lifted" directly from the underlying relational schema using an existing unsupervised approach. Finally, we apply our approach to three online databases to extract RDF data. Our results indicate that this approach can be used to bootstrap and speed up the migration of life science data into the Linked Open Data cloud.
\end{abstract}

\section{Introduction}

The Life Sciences have seen a data explosion in the last decade. These data are most commonly stored in freely-accessible Web-based databases. The Nucleic Acids Research (NAR) Journal puts the current number of such Web databases that also have an application note with the journal at 1170 [1. As the number of these databases increases, so does their size. Large Proteomics and Genomics databases actually exhibit exponential growth. These databases often contain complementary data, pertaining to narrow and specialized sub-domains. Any meaningful scientific investigation typically requires accessing many sources, manually extracting and linking data records together. This activity is made even more difficult with the fact that search engines cannot index most dynamically

L. Aroyo et al. (Eds.): ESWC 2010, Part II, LNCS 6089, pp. 319 333, 2010.

(C) Springer-Verlag Berlin Heidelberg 2010 
generated Web pages. Therefore, there is a pressing need for providing unified and integrated access to these sources.

Traditional database integration techniques require direct access to the underlying relational database. However, in the scenario presented above, this is almost never the case. In some instances, a part of the database is made available for download in diverse formats, including XML, tab-delimited text files, spreadsheets, or proprietary formats. Furthermore, these data can be often stale and incomplete.

More recently, there have been a number of approaches using Semantic Web technologies to create RDF triple stores by aggregating data from various Biochemical databases. The earliest approach was YeastHub [2, which built a centralized RDF store for Yeast data collected from eight online sources. The data was mapped on to a manually built ontology, as well as existing ontologies like RSS1 and Dublin Cor². In FungalWeb [3], an OWL-DL ontology was manually developed and instantiated for describing enzymatic data. Stephens et al. 4 provided a drug-discovery use case by manually inspecting and integrating 12 biochemical databases into an RDF data model. Similarly, Pasquier [5] integrated more than 10 life science databases by manually creating an ontology and merging it with existing ontologies like $\mathrm{GO}^{3}$ and $\mathrm{GOA}^{4}$. FlyWeb 6 integrates textual and image data for Drosophila from three databases, using the D2RQ [7] tool to convert relational data into RDF, as well as hand-written scripts for spreadsheet data. Bio2RDF [8] is perhaps the largest source of Biochemical RDF data. It integrates information from a variety of formats using hand-written "RDFizer" programs that populate an OWL ontology that has been manually created. The SBMM toolbox [9] shares a feature with Bio2RDF, whereby wrappers are manually created for specific source database Web sites. A user query is transmitted to search interfaces of appropriate sources, and the wrappers extract and convert the generated data into RDF.

One common limiting factor in the above mentioned approaches is the significant amount of manual work required to construct ontologies, populate data from legacy formats into this ontology and to link this data. We address the first two challenges by proposing unsupervised techniques for ontology learning and data extraction. We begin by answering the following two basic questions:

1. What sources should we utilize for ontology learning?

2. Which data format should we target for automatic data extraction?

In an ideal world, all sources would provide their data directly in RDF format. However, only a handful of sources, such as UniProt, Gene Ontology, IntAct and NCBI Taxonomy, do so. The wide variety of export formats make the task of unsupervised learning extremely hard and in many cases there is no possibility to export data at all. Furthermore, Biochemical databases are updated

\footnotetext{
${ }^{1}$ http://web.resource.org/rss/1.0/

${ }^{2}$ http://dublincore.org/

${ }^{3}$ http://www.geneontology.org/

${ }^{4}$ http://www.ebi.ac.uk/GOA/
} 
very frequently, rendering the exported data stale and outdated, as mentioned above. Finally, we conducted a survey 5 of 100 databases listed on the NAR Journal to assess how wide-spread the adoption of direct programmatic access to databases was, such as through Web Service APIs. We discovered that only 11 large and well-established sources actually provide complete or partial access through APIs. In fact, we concluded that a Web interface was the only common access point for all databases.

In this paper, we propose to use Web interfaces of Biochemical sources to learn corresponding ontologies. This follows our previous work [10] on unsupervised wrapper generation for entire Biochemical Web sites to extract labeled data from multiple classes of pages. Together, these approaches can be used to automatically acquire ontologies and instantiate them with RDF data on-the-fly. We argue that such an approach would at least help to bootstrap the process which all of the projects mentioned above follow: that of converting Web-accessible data into linked RDF data. Additionally, our approach can be used to learn domain-specific ontologies, a task that is very challenging if performed on natural language text as opposed to labeled, (semi) structured data in our setting. This is very much in line with the vision of the self-annotating Web [1] where the Web is used to extract ontologies, which then provide semantic annotation to enable Semantic Web content creation.

Our approach to ontology learning relies on a set of transformation rules that we apply to the output of our wrapper-generation algorithms, resulting in an OWL ontology for the underlying database. We apply our algorithms to realworld online Biochemical Web sites. We perform a "hard" evaluation of our acquired ontologies against gold standard ontologies, and a "soft" evaluation using three human experts to rate T-Box statements, or axioms, from our ontologies. We also evaluate our ontologies against ontologies that are lifted directly from the relational schemata. Finally, we apply our approach to extract RDF data from three Web sources.

The rest of this paper is organized as follows. Section 2 briefly explains our wrapper generation algorithm and its output which is used for ontology learning. Section 3 presents our transformation rules which convert our wrapper algorithm's output into an ontology, while Section 4 presents the experiments that we conducted for ontology learning and their evaluations. Section 5 briefly describes the extraction of RDF data. Section 6 presents the related work, and we conclude in Section 7.

\section{Wrapper Induction}

This section presents a brief overview of our wrapper induction approach to extract labeled data from Biochemical Web sites. A complete description is available in [10].

\footnotetext{
${ }^{5}$ Available at http://sabiork.villa-bosch.de/ontology/servicesurvey.html
} 


\subsection{Page-Level Wrapper Induction}

We observe that data on Biochemical Web sites are often labeled - that is, data entries on the Web pages are in proximity to descriptive text which serve the purpose of attribute names, such as "Mass" and "Temperature". Our survey6 of 20 such Web sites revealed that, in fact, about $97 \%$ of data fields present on these Web pages were labeled. We utilize these labels to by-pass the page structure, which is dynamic and hence unpredictable, and pivot directly to the labels, and find a relative path in the DOM representation of the page to the corresponding data.

Our algorithm relies on multiple sample pages belonging to the same class that is, having similar structure and content, and being generated from the same server-side script. We screen-scrape the individual text entries from each sample, and compare these entries across all samples. Disjoint entries are classified as data entries, where overlapping entries are a mixture of presentation text and possible labels. We then determine XML Path expressions (XPath expressions) for each text entry, from the root of the DOM tree to the node containing that entry. These XPaths are used to determine, for each data entry, the closest nondata entry, which serves as its label. Ultimately, our algorithm outputs a label with a corresponding relative XPath to the corresponding data entries. A final XPath expression resembles the form:

\section{$/ / *[\operatorname{text}()=$ 'label']/. / tr $[1] / \operatorname{td}[2] / \mathrm{a}[1] / \operatorname{text}()$}

The above XPath expression reads: Jump to the node which contains the text "label", follow a relative path in the DOM tree from this node, which points to the corresponding data. Our experiments indicated that the algorithm achieves a Precision of $99 \%$ and a Recall of $98 \%$ with about 9 samples.

\subsection{Site-Wide Wrapper Induction}

Data in Life Science Web sites are often scattered across many pages belonging to many different classes. In order to extract all data from underlying databases, we must learn wrappers for each of these classes. We tackle this problem using the concept of Labeled Link Collections. Link collections are hyperlink tag(s) which appear grouped together under the same parent node. A labeled link collection implies a link collection that has been associated with a label by our algorithm in Section 2.1, signifying these hyperlinks occur over data fields. We make two crucial observations about labeled link collections. Firstly, such collections point to data-rich pages, and secondly, all target pages of a labeled link collection belong to the same class. The latter observation allows us to automatically provide pages that have similar structure and content to our pagelevel wrapper induction algorithm.

The site-wide wrapper induction algorithm proceeds by learning a wrapper for the initial result page generated from probing a search form. It finds labeled link

\footnotetext{
${ }^{6}$ Available at http://sabiork.villa-bosch.de/ontology/labelsurvey.html
} 


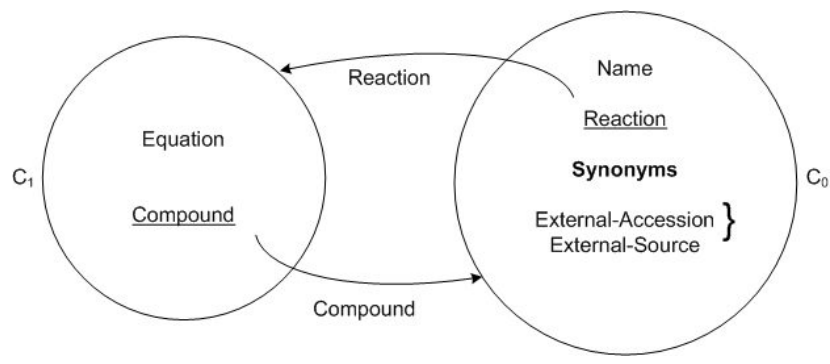

Fig. 1. Schematic representation of wrapper induction output

collections and follows them to their target pages, learning wrappers for those pages. Upon learning a new wrapper, it compares this new wrapper with existing wrappers by comparing the sequence of corresponding labels. If the sequence is the same, it implies the link collection points towards pages of the same class. This process is iterated for each new wrapper learnt, until data-rich pages of a Web site are explored.

This process essentially results in a Web site model as a directed labeled graph, schematically shown in Figure 1, discussed in more detail in Section 2.3. Experiments showed that the site-wide wrapper induction algorithm achieves a Precision of about $98 \%$ and a Recall of $76 \%$.

\subsection{Discussion of Wrapper Output}

In this section, we discuss in more detail the output of our site-wide wrapper induction algorithm. The actual output is in XML, but is shown schematically in Figure 1, for a hypothetical source. The circles represent a class of pages, while their contents are the labels that were identified by our wrapper in these pages, together with XPaths of corresponding data (not shown). These label-data pairs have some additional meta-data associated with them. Certain label-data pairs have hyperlink tags on them, signifying that these are link collections (underlined in the figure), others have many data values associated with a single label (shown in bold) as opposed to some which have a single data value (normal font), while others are classified as composites (shown connected together). In addition, the figure also shows directed edges between classes of pages. For a given class of pages, an edge directed outwards represents the virtual link with a class of pages that can be reached when following a particular link collection. In Figure 1, the link collection "Reaction" in class $C_{0}$ when followed, leads to a class of pages $C_{1}$. The edge signifying this directed connection is named after the corresponding link collection "Reaction".

\section{Ontology Learning}

In this section we describe our transformation rules to convert the output presented in Section 2 into an ontology. We select OWL as the ontology language as 
it is being used in current Semantic Web projects in the Life Science mentioned in Section 1. Finally, all the information required to determine the application of these rules is present in our wrapper output. Therefore, no supervision is required to apply these rules. In the subsequent sub-sections, we take Figure 1 as a running example and explain our transformation rules for constructing classes, data properties and object properties of an ontology.

\subsection{Transformation Rules for Classes}

We apply the following two rules for construction and naming of classes:

$C C$ - Class Construction: Each page-class discovered by our wrapper induction algorithm is converted into a class in our ontology. This is based on our observation that a Web page typically represents a concept. In our example, this rule would construct two classes corresponding to the two page-classes discovered. In our example, two classes will be constructed corresponding to the two page-classes present.

$C N$ - Class Naming: A class is named after the label of the link collection that points to it. The class corresponding to $C_{1}$ will be named "Reaction".

\subsection{Transformation Rules for Properties}

DP - DataType Property Construction: All label-data pairs discovered within a page-class that do not have hyperlink tags are converted into data type properties. The domain of these properties is the OWL class which corresponds to the page-class which contains these data-label pairs, and a suitable range is selected to describe the data. Currently, our implementation supports the XML Schema types string, integer and float. The choice between these types is based on a simple string parsing to determine whether the sample data contains characters, digits or decimals.

$O P$ - Object Property Construction: All label-data pairs discovered within a page-class that have hyperlink tags are converted into object properties. The domain of these properties is the OWL class which corresponds to the pageclass which contains these data-label pairs where the range is the target class to which the link collection points.

$P N$ - Property Naming: The label of the corresponding data-label pair is assigned as the name of the property.

PF - Setting a Property to Functional: Labels discovered in the page-classes that have single data values associated with them are set as functional. This can be determined by examining the XPath expressions as described in Section 2.1. For example, for the Reaction class in Figure 1, we have the data type property

Equation(Reaction, XSDString)

An example of an object property is

_Compound(Reaction, Compound) 


\subsection{Transformation Rules for Composite Labels}

Labels which appear in adjoining columns of a table have associated data values that fully describe their semantics when viewed together, which signifies an nary relation. Instead of constructing properties from these labels as shown in Section 3.2, we create an object property which has in its range a new class. This new class in turn is used to create functional properties from label-data pairs as shown in Section 3.2. We define the following rules to achieve this:

CL.a - Class Construction from Composite Labels: A class is created in the ontology for every set of composites labels.

CL.b - Naming of Class Created from Composite Labels: Labels are concatenated to form the name of such classes. In our running example, a class will be created with the name "External_Accession-External_Source".

CL.c - Object Property Creation: An object property is created which has a range of this new class created, and a domain of the OWL class which corresponds to the page-class which contains these data-label pairs.

Finally, transformation rules $D P$ and $O P$ are applied to create properties in the same manner as described in Section 3.2, except the domain of these properties is the new class that has been created by rule CL.a. The transformations described in this sub-section are applied for each set of composite label-data pairs that are identified by our wrapper induction algorithm.

\section{Experiments and Evaluation}

This section describes our experiments on 13 real-world Biochemical Deep Web sources, and the evaluation of the results we obtained. We implemented the transformation rules described in Section 3 in a Java program that accepts the XML output of our wrapper induction algorithms and applies these rules in an unsupervised manner to construct an OWL ontology.

The 13 online Biochemical databases that were targeted for ontology learning are presented in Table 1, together with the number of classes, object and data type properties discovered by our approach. These sources were selected as they provide basic qualitative data that is widely required in most specialized domains and are extensively used for annotation purposes by various other Web sites and in models. As Table 1 indicates, the number of classes discovered varies. In our wrapper-induction approach detailed in [10], each page type which describes data is converted into a class. The greater the number of pages across which the results are distributed, the greater will be the number of classes in the learned ontology. (In addition, some classes may also be introduced in the resulting ontology due to the transformation rules for composite labels, as described in Section 3.3). For instance, Rhea and SBO are relatively small databases and display all their results on a single result page. Therefore, the corresponding ontologies contain only a single class. On the other hand, Reactome and UniProt are large databases 
Table 1. Targeted data sources \& corresponding classes \& properties of learned ontologies

\begin{tabular}{|l|l|l|l|}
\hline Database & Classes & Object Properties & Data Properties \\
\hline Reactome http://www.reactome.org/ & 17 & 31 & 42 \\
\hline PubChem http://pubchem.ncbi.nlm.nih.gov & 10 & 16 & 61 \\
\hline Rhea http://www.ebi.ac.uk/rhea/ & 1 & 1 & 5 \\
\hline PDB (USA) http://www.rcsb.org/ & 8 & 12 & 55 \\
\hline PDBe http://www.ebi.ac.uk/pdbe/ & 3 & 3 & 21 \\
\hline UniProt http://www.uniprot.org/ & 12 & 15 & 68 \\
\hline KEGG http://www.genome.jp/kegg/ & 7 & 18 & 26 \\
\hline IntAct www.ebi.ac.uk/intact/ & 7 & 9 & 23 \\
\hline SABIO-RK http://sabio.villa-bosch.de/ & 18 & 22 & 46 \\
\hline ChEBI http://www.ebi.ac.uk/chebi/ & 8 & 9 & 24 \\
\hline SBO http://www.ebi.ac.uk/sbo/ & 1 & 1 & 8 \\
\hline IntEnz http://www.ebi.ac.uk/intenz/ & 1 & 0 & 10 \\
\hline MSDChem http://ebi.ac.uk/msd-srv/chempdb/ & 1 & 1 & 22 \\
\hline
\end{tabular}

with the results spread across many different types of pages. This fact, together with the presence of composite labels, results in many classes in corresponding ontologies. We perform three evaluations for a selection of the ontologies that we learn. Firstly, we perform a "hard" evaluation against a gold standard ontology. This evaluation covers the lexical term layer and the concept hierarchy evaluation. The second "soft" evaluation is done with the help of domain experts which evaluate T-Box axioms from our ontologies in order to evaluate data and object properties (relations), as well as class and relation names. Thirdly, we "lift" ontologies from actual relational schemata, where available, using an exiting unsupervised approach. We then ask domain experts to rate T-Box axioms from these "lifted" ontologies against corresponding ontologies that are learned using our approach. These evaluations are presented subsequently in Sections $4.2,4.3$ and 4.4 respectively. Section 4.1 briefly describes the process of obtaining gold-standard ontologies for the three target sources. We provide an analysis of our results in Section 4.5, highlighting the limitations of our approach.

\subsection{Acquiring Gold Standard Ontologies}

As mentioned above, we are interested in evaluating T-Box style axioms from our learned ontologies. (Note that the evaluation of the extracted data has already been performed in [10] for our wrapper-induction algorithm). In order to do this, we need corresponding T-Box style gold standard ontologies. However, existing relational databases rarely, if at all, provide corresponding ontologies.

In the Bio2RDF project a global ontology describing many namespaces corresponding to various data sources was manually developed7. We extract the

$\overline{7}$ Available at http://bio2rdf.org/bio2rdf.owl 
classes and relations in the KEGG and PDB namespace from this global ontology to use as our gold standard. In case of ChEBI and SBO, only A-Box style ontologies containing assertions about individuals are available in various formats, including OWL. We manually reverse engineer a T-Box from these ontologies to use as gold standard for these two sources respectively.

\subsection{Evaluation against a Gold Standard}

We use the framework described by Dellschaft et al. 12 to evaluate a learned ontology against a gold standard. We provide a brief overview of the evaluation measures here, and refer an interested reader to [12 for details. They provide measures for evaluating the lexical term layer and taxonomy of an ontology. For lexical evaluation, these measures include lexical precision and lexical recall, borrowed from [13] and defined as:

$$
L P\left(O_{C}, O_{R}\right)=\frac{\left|C_{C} \cap C_{R}\right|}{\left|C_{C}\right|} \quad, \quad L R\left(O_{C}, O_{R}\right)=\frac{\left|C_{C} \cap C_{R}\right|}{\left|C_{R}\right|}
$$

Where $O_{C}$ and $O_{R}$ are the computed and reference ontologies respectively, and $C_{C}$ and $C_{R}$ are concepts in these ontologies, identified by their names.

For taxonomic evaluation, they provide precision and recall measures which are based on using the common semantic cotopy as the characteristic extract of concepts in a taxonomy. A characteristic extract of a concept characterizes the position of a concept in a hierarchy, which can be used to determine local taxonomic precision for that concept. This in turn is used as a building block for global taxonomic evaluation of the ontology. By advising to use the common semantic cotopy, Dellschaft et al. effectively diminishing the influence of the lexical layer in the evaluation of the taxonomy, by removing concepts which are (lexically) different from the semantic cotopy, and only including common concepts in the semantic cotopy. The taxonomic precision and recall are, thus, defined as

$$
\begin{gathered}
T P_{S C}\left(O_{C}, O_{R}\right):=\frac{1}{\left|C_{C}\right|} \sum_{c \in C_{C}}\left\{\begin{array}{cc}
t p_{s c}\left(c, c, O_{C}, O_{R}\right) & \text { if } c \in C_{R} \\
0 & \text { if } c \notin C_{R}
\end{array}\right. \\
T R_{S C}\left(O_{C}, O_{R}\right):=T P_{S C}\left(O_{R}, O_{C}\right)
\end{gathered}
$$

Where

$$
t p_{s c}\left(c_{1}, c_{2}, O_{C}, O_{R}\right):=\frac{\left|c e\left(c_{1}, O_{C}\right) \cap c e\left(c_{2}, O_{R}\right)\right|}{\left|c e\left(c_{1}, O_{C}\right)\right|}
$$

is the local taxonomic precision of a concept. The F-measures are then given by:

$$
T F\left(O_{C}, O_{R}\right):=\frac{2 \cdot T P\left(O_{C}, O_{R}\right) \cdot T R\left(O_{C}, O_{R}\right)}{T P\left(O_{C}, O_{R}\right)+T R\left(O_{C}, O_{R}\right)}
$$




$$
T F^{\prime}\left(O_{C}, O_{R}\right):=\frac{2 \cdot L R\left(O_{C}, O_{R}\right) \cdot T F\left(O_{C}, O_{R}\right)}{L R\left(O_{C}, O_{R}\right)+T F\left(O_{C}, O_{R}\right)}
$$

Where $T F^{\prime}$ combines the lexical level and taxonomic evaluation in a single value.

We apply these measures on our learned ontologies comparing them to reference ontologies obtained in Section 4.1. The results are presented in Table 2.

Table 2. Results of evaluation against reference ontologies

\begin{tabular}{|l|l|l|l|l|l|l|}
\hline & $L P$ & $L R$ & $T P$ & $T R$ & $T F$ & $T F^{\prime}$ \\
\hline KEGG & 0.428 & 0.428 & 0.428 & 0.357 & 0.39 & 0.4 \\
\hline ChEBI & 0.13 & 1.0 & 0.125 & 1.0 & 0.22 & 0.36 \\
\hline SBO & 0.5 & 1.0 & 0.5 & 1.0 & 0.6 & 0.8 \\
\hline PDB USA & 0.13 & 1.0 & 0.125 & 1.0 & 0.22 & 0.36 \\
\hline
\end{tabular}

Discussion: The lexical and taxonomic recall for our approach in case of ChEBI, $\mathrm{SBO}$ and PDB in Table 2 are perfect. This is because we are successfully able to discover the classes in the gold standard ontologies. The lower precision is explained by the fact that we construct additional classes ( 7 in the case of ChEBI and PDB, 1 in the case of SBO) in our ontologies. However, in the case of ChEBI and SBO all of these classes are constructed to express n-ary relationships between composite attributes that we discover during the wrapper induction phase. Therefore, although our approach strives to maintain the semantics of the data, it suffers with regard to the taxonomic precision due to this. In the case of PDB, the Bio2RDF ontology omits certain data offered by this source. Hence the gold standard ontology contains fewer classes than our approach can discover from the Web interface, resulting in lower precision. The results for KEGG are mixed; there are some classes we discover correctly, while others we are not able to discover. This is indicated by the relatively equal precision and recall values for both the lexical and taxonomic results. It should be mentioned that these results are quite comparable to human inter-ontology building [28].

\subsection{Evaluation by Domain Experts}

The evaluation measures in Section 4.2 have served to evaluate the names and taxonomy of classes. They do not address the data and object properties that are defined for these classes. For scientific data, especially Life Science data, this is crucial, as entities in such domains have rich relationships with each other. In this section, we describe the results of a "soft" evaluation, where we ask domain experts to rate axioms in our ontologies in order to judge how well we were able to determine these relations. These T-Box statements are of the following form

Synonym(Gene, XSDString)

Class RPair 
We ask three domain experts to rate 51 such statements from our KEGG ontology, 41 from ChEBI, 11 from SBO and 54 statements from our PDB ontology. We allow them to refer to corresponding Web sites, so that they can compare our findings against what they would have selected manually from these sites. We ask our experts to classify these statements into four categories based on the degree of correctness: Wrong, Slightly Correct, Mostly Correct, and Correct. They are instructed to denote these categories for each statement by assigning values of $0,1,2$ and 3 respectively. We then use these ratings to determine our precision and recall at three distinct points. First, using our experts' ratings we determine the categorical agreement between them. We calculate the Kappa statistic from the ratings of each ontology, using the method described in 14 for multiple raters rating into multiple categories. The Kappa values were measured to be $42.24 \%, 27.42 \%$, $13.10 \%$ and $47.31 \%$ for KEGG, ChEBI, SBO and PDB respectively, which indicates that there is a low agreement. In addition, Kappa values generally tend to decrease as the number of categories increases, as in our case. The low agreement might also suggest that the ontology construction task is inherently hard or not well defined. For our evaluation, we count the data points where all three raters agree on the categorization of a given statement from our ontology, and determine the precision for each category. Our true-positives for a given categorization are the number of statements unanimously classified into this category by our raters. The false-positives are the number of statements unanimously classified into some other category. Therefore, we determine precisions for Correct (C), Mostly Correct (MC) and Slightly Correct (SC) statements, with results shown in Table 3. For completely correct statements, we have an average precision of $53.25 \%$. There can be different factors for this, such as incorrect discovery of label-data pairs by our wrapper induction algorithm, or incorrect or unsuitable assignment of class and property names (Recall from Sections 3.2 and 3.3 that names are taken from corresponding labels of link-collections).

\subsection{Evaluation against Lifted Ontologies}

We are interested in evaluating the results of our approach to extracting ontologies from Web interfaces against ontologies that are "lifted" from direct access to the underlying relational schema using existing unsupervised approaches such as [18] or 19], which apply transformation rules to a relational schema and reverse-engineer an ontology. We opt to use the approach described in [19] as it constructs ontologies in OWL. However, from our selection of 13 databases, we are only able to get direct access to the relational schemata for two sources, namely SABIO-RK and ChEBI. We therefore lift ontologies from these schemata and perform a manual evaluation of our learned ontologies with the help of a domain expert. We provide sets of 20 corresponding statements from the lifted and learned ontologies, and instruct the expert to give a score of 1 to the superior statement and a score of 0 to the poorer statement, taking into consideration both syntax and semantics. (A score of 1 is given to both statements if they are deemed equally valid). The results are shown in Table 4, which presents the total score for corresponding lifted and learned ontologies. 
Table 3. Precision values for three categorizations of statement. $\mathrm{P}(\mathrm{C})$ is precision of statement being completely correct, $\mathrm{P}(\mathrm{MC})$ is precision of statement either completely or mostly correct, $\mathrm{P}(\mathrm{SC})$ is precision of statement completely, mostly or slightly correct.

\begin{tabular}{|l|l|l|l|}
\hline Sources & $\mathrm{P}(\mathrm{C})$ & $\mathrm{P}(\mathrm{MC})$ & $\mathrm{P}(\mathrm{SC})$ \\
\hline KEGG & $43.8 \%$ & $84.4 \%$ & $90.7 \%$ \\
\hline ChEBI & $66.6 \%$ & $80.1 \%$ & $88.4 \%$ \\
\hline SBO & $50 \%$ & $87.5 \%$ & $87.5 \%$ \\
\hline PDB USA & $52.6 \%$ & $90.5 \%$ & $92.3 \%$ \\
\hline
\end{tabular}

Table 4. The total score for lifted and learned ontologies

\begin{tabular}{|l|l|l|}
\hline Sources & Total Score for Lifted Ontology & Total Score for Learned Ontology \\
\hline SABIO-RK & 9 & 16 \\
\hline ChEBI & 19 & 14 \\
\hline
\end{tabular}

Discussion: We note here that for SABIO-RK, 34 classes are constructed in the ontology lifted directly from the relational schema compared to the 18 classes constructed using our approach. This is because portions of the schema are not normalized, as well as the fact that the schema contains certain data which is not displayed in the Web interface at all. The ontology lifted from this schema also suffers lexically as the table and attribute names are often obscure and unnatural. On the other hand, both the ontologies for ChEBI consist of 8 classes, and the schema comprises of well-named tables and attributes, resulting in a very high score for the lifted ontology.

\subsection{Analysis of Results}

In this sub-section we present a brief analysis of the overall results and the conclusions that we can derive from it. Firstly, our transformation rules do not assist us in creating rich taxonomies. Therefore, we would expect to achieve poor taxonomic precision and recall if the reference ontology has a rich taxonomy, which was not the case in our experiments: Only KEGG has two sub-class relationships. A possible future improvement in our approach would be to use labels within page-classes in our wrapper output to determine such relations. One could determine such taxonomic relations if the set of labels of a given class is a subset of that of another class, for example, by using Formal Concept Analysis [27.

Secondly, our results from Section 4.4 indicate that extraction of ontologies from Web interfaces is a viable option especially in the absence of relational schemata. Relational schemata often suffer from not being in normalized forms, either for ease of representation or performance, and may follow ambiguous naming conventions as these are private and internal representations.

Finally, the manual approaches for ontology construction described in Section 1 tend to re-use existing ontologies, such as FOAF 8 and Dublin Core.

\footnotetext{
${ }^{8}$ http://www.foaf-project.org/
} 
This is extremely beneficial, as it helps to eventually integrate the data from various sources. However, this is not possible in our approach. This results in lexical differences between manually constructed ontologies and those generated by using our approach, which affects precision and recall.

\section{RDF Data Extraction}

In this section we briefly describe extraction of RDF triples from Web interfaces for which we have learned corresponding ontologies. Since the transformation rules for our ontology learning approach are directly mapped from the output of our wrapper induction algorithm in 10, we can directly extract RDF data by applying our wrappers and populating the corresponding ontology. Since we wish to merely demonstrate the feasibility of extracting data from Web pages, we select three sources of varying size, namely KEGG, ChEBI and MSDChem, and execute corresponding wrappers on the result pages. We utilize the lists of identifiers provided by each source on its Web site to probe the Web forms and generate the result pages. The triples thus generated are stored in a local Sesame 9 repository. In all, approximately $300 \mathrm{MB}, 200 \mathrm{MB}$ and $25 \mathrm{MB}$ of data are extracted for KEGG, ChEBI and MSDChem respectively. The running times for applying the wrappers for these sources were approximately 7, 5 and 1 hours respectively, using a $6 \mathrm{Mbps}$ internet connection.

\section{Related Work}

Although there are many approaches to ontology learning from natural language text, we are only aware of a handful of approaches which target Deep Web sites containing semi-structured data as a source for ontology learning. In [15], the authors describe a system which extracts attributes of Web search forms belonging to a certain domain, such as tourism and e-commerce. WordNet is used to find hyponyms for these attributes iteratively until a taxonomy of concepts is generated using only the IS-A relation. OntoBuilder [16] also constructs a taxonomy of concepts by parsing Web search forms, although the authors do not describe the algorithm or the resulting taxonomy in detail. OntoMiner [17] learns a taxonomy of concepts from co-domain Web sites. It relies on HTML regularities to construct taxonomic structures in XML by utilizing a hierarchical partition algorithm. The taxonomy is iteratively expanded with sub-concepts by crawling through links corresponding to concepts in the taxonomy. Another related approach is used in Triplify 29], which facilitates linked RDF data generation for Web site publishers by mapping HTTP-URI requests onto relational queries, the results of which are transformed into RDF.

A closely related area of research is Database-to-ontology conversion, whereby transformation rules similar to ours are applied directly to a relational database schema and corresponding ontology is extracted [1819], or mapping rules are generated between an existing ontology and the underlying relational database,

${ }^{9}$ http://www.openrdf.org/ 
such as in D2RQ [7. However, in our setting, we do not have access to the actual database implementations. Another related research topic is that of identification of HTML tables and transforming them into, for instance, logical frames as in TARTAR 20 or relational schemes as in WebTables 21. However, such approaches neglect data on Web pages that are not structured within HTML tables. Finally, there has been considerable research using XML documents to extract relational schema [22 23], DTDs [24], or XML Schemata 25 26].

\section{Conclusions}

We have presented an unsupervised approach for extracting ontologies and RDF data from Deep Web Life Science databases. Our approach relies on transformation rules that convert XML output from our wrapper induction algorithm into OWL ontologies. Experiments were conducted on real-world online Biochemical Web sites. Our results indicate that this approach can be used to bootstrap the process of converting legacy and freely available online data into machineprocesable data for use in Semantic Web applications. Our results support our argument that the Deep Web, with its semi-structured content, is an ideal source for learning ontologies to help overcome the bottleneck for wider adoption of Semantic Web technologies. For future work, we would like to re-use existing ontologies during the ontology learning phase in order to facilitate the eventual integration of the extracted data into the Linked Open Data cloud.

\section{References}

1. Galperin, M.Y., Cochrane, G.R.: Nucleic Acids Research annual Database Issue and the NAR online Molecular Biology Database Collection in 2009. Nucleic Acids Res. 37(Database issue), 1-4 (2009)

2. Cheung, K.H., et al.: YeastHub: a semantic web use case for integrating data in the life sciences domain. Bioinformatics 21(suppl. 1) (June 1, 2005)

3. Baker, C.J.O., et al.: Semantic Web infrastructure for fungal enzyme biotechnologists. Journal of Web Semantics 3(4) (2006)

4. Stephens, S., LaVigna, D., Dilascio, M., Luciano, J.: Aggregation of bioinformatics data using semantic web technology. Journal of Web Semantics, 4 (2006)

5. Pasquier, C.: Biological data integration using Semantic Web technologies. Biochimie 90(4), 584-594 (2008)

6. Zhao, J., Miles, A., Klyne, G., Shotton, D.: OpenFlyData: The Way to Go for Biological Data Integration. In: Paton, N.W., Missier, P., Hedeler, C. (eds.) Data Integration in the Life Sciences. LNCS (LNBI), vol. 5647, pp. 47-54. Springer, Heidelberg (2009)

7. Bizer, C.: D2RQ - treating non-RDF databases as virtual RDF graphs. In: Proceedings of the 3rd International Semantic Web Conference ISWC 2004 (2004)

8. Belleau, F., et al.: Bio2RDF: Towards a mashup to build bioinformatics knowledge systems. In: WWW 2007, Banff, Canada (2007)

9. Reyes-Palomares, A., et al.: Systems Biology Metabolic Modeling Assistant (SBMM): An ontology-based tool for the integration of metabolic data in kinetic modeling. Bioinformatics, doi:10.1093/bioinformatics/btp061 
10. Mir, S., Staab, S., Rojas, I.: Site-Wide Wrapper Induction for Life Science Deep Web Databases. In: Paton, N.W., Missier, P., Hedeler, C. (eds.) DILS 2009. LNCS, vol. 5647, pp. 96-112. Springer, Heidelberg (2009)

11. Cimiano, P., Handschuh, S., Staab, S.: Towards the Sself-Annotating Web. In: WWW 2004: Proceedings of the 13th International Conference on World Wide Web, pp. 462-471. ACM Press, New York (2004)

12. Dellschaft, K., Staab, S.: On How to Perform a Gold Standard based Evaluation of Ontology Learning. In: Cruz, I., Decker, S., Allemang, D., Preist, C., Schwabe, D., Mika, P., Uschold, M., Aroyo, L.M. (eds.) ISWC 2006. LNCS, vol. 4273, pp. 228-241. Springer, Heidelberg (2006)

13. Sabou, M., Wroe, C., Goble, C., Mishne, G.: Learning domain ontologies for web service descriptions: an experiment in bioinformatics. In: Proc. of WWW 2005 (2005)

14. Green, A.M.: Kappa statistics for multiple raters using categorical classifications. In: Proceedings of the Twenty-Second Annual Conference of SAS Users Group (1997)

15. An, Y.J., et al.: Automatic Generation of Ontology from the Deep Web. In: DEXA Workshops 2007, pp. 470-474 (2007)

16. Roitman, H., Gal, A.: OntoBuilder: Fully Automatic Extraction and Consolidation of Ontologies from Web Sources using Sequence Semantics. In: Proceedings of the International Conference on Semantics of a Networked World, ICSNW 2006 (2006)

17. Davalcu, H., Vadrevu, S., Nagarajan, S., Ramakrishnan, I.: Ontominer: bootstrapping and populating ontologies from domain-specific web sites. IEEE Intelligent Systems 18(5), 24-33 (2003)

18. Stojanovic, L., Stojanovic, N., Volz, R.: Migrating data-intensive Web Sites into the Semantic Web. In: Proc. of the 17th symposium on Proceedings of the 2002 ACM Symposium on Applied Computing, SAC 2002, Madrid Spain, pp. 1100-1107 (2002)

19. Li, M., Du, X.-Y., Wang, S.: Learning ontology from relational database. In: Proceedings of International Conference on Machine Learning and Cybernetics (2005)

20. Pivk, A., et al.: Transforming arbitrary Tables into F-Logic Frames with TARTAR. Data \& Knowledge Engineering (DKE) 60(3), 567-595 (2007)

21. Cafarella, M.J., et al.: Uncovering the relational Web. In: WebDB 2008 (2008)

22. Deutsch, A., Fernandez, M., Suciu, D.: Storing Semistructured Data in Relations. In: Proceedings of the Workshop on Query Processing for Semistructured Data and Non-Standard Data Formats (1998)

23. Cohen, S., Kanza, Y., Sagiv, Y.: Generating Relations from XML Documents. In: Calvanese, D., Lenzerini, M., Motwani, R. (eds.) ICDT 2003. LNCS, vol. 2572, pp. 282-296. Springer, Heidelberg (2002)

24. Garofalakis, M.N., et al.: DTD inference from XML documents: The xtract approach. IEEE Data Eng. Bull. 26(3), 19-25 (2003)

25. Hegewald, J., Naumann, F., Weis, M.: XStruct: Efficient Schema Extraction from Multiple and Large XML Documents. In: Data Engineering Workshop, 22nd International Conference on Data Engineering Workshops (ICDEW 2006), p. 81 (2006)

26. Bex, G.J., Neven, F., Vansummeren, S.: Inferring XML schema definitions from XML data. In: VLDB 2007, pp. 998-1009 (2007)

27. Cimiano, P., Hotho, A., Staab, S.: Learning Concept Hierarchies from Text Corpora using Formal Concept Analysis. J. Artif. Intell. Res. (JAIR) 24, 305-339 (2005)

28. Maedche, A., Staab, S.: Measuring Similarity between Ontologies. In: Gómez-Pérez, A., Benjamins, V.R. (eds.) EKAW 2002. LNCS (LNAI), vol. 2473, p. 251. Springer, Heidelberg (2002)

29. Auer, S., et al.: Triplify - Light-Weight Linked Data Publication from Relational Databases. In: 18th International World Wide Web Conference, pp. 621-621 